\title{
Relación entre actitudes hacia el endeudamiento y discrepancia del yo en estudiantes de pedagogía chilenos
}

Relation between attitudes towards the indebtedness and self-discrepancy in chilean pedagogy students

\section{Volumen 17, Número 3}

Setiembre-Diciembre

pp. 1-28

\author{
Este número se publica el $1^{\circ}$ de setiembre de 2017 \\ DOI: http://dx.doi.org/10.15517/aie.v17i3.29248 \\ Marianela Denegri Coria \\ Claudia Caro Vallejos \\ María Isabel Cerda Figueroa \\ Katya Eschmann Gacitúa \\ Nathaly Martínez Guaiquil \\ Jocelyne Sepúlveda Aravena
}

Revista indizada en REDALYC, SCIELO

Revista distribuida en las bases de datos:

LATINDEX, DOAJ, REDIB, IRESIE, CLASE, DIALNET, SHERPA/ROMEO, QUALIS-CAPES, MIAR

Revista registrada en los directorios:

ULRICH'S, REDIE, RINACE, OEI, MAESTROTECA, PREAL, CLACSO 


\title{
Relación entre actitudes hacia el endeudamiento y discrepancia del yo en estudiantes de pedagogía chilenos
}

Relation between attitudes towards the indebtedness and self-discrepancy in chilean pedagogy students

\author{
Marianela Denegri Coria ${ }^{1}$ \\ Claudia Caro Vallejos ${ }^{2}$ \\ María Isabel Cerda Figueroa ${ }^{3}$ \\ Katya Eschmann Gacitúa ${ }^{4}$ \\ Nathaly Martínez Guaiquil ${ }^{5}$ \\ Jocelyne Sepúlveda Aravena ${ }^{6}$
}

\begin{abstract}
Resumen: La globalización ha trastocado diferentes normas y dinámicas sociales, dando énfasis a prácticas asociadas al consumo y al endeudamiento. En el presente artículo, resulta de particular interés el segmento de jóvenes universitarios estudiantes de pedagogía debido a que; por un lado, son ciudadanos activos y a la vez son responsables de la formación de nuevos ciudadanos. En este marco, este estudio pretendió establecer la asociación entre variables demográficas, discrepancia del yo y la actitud hacia el endeudamiento en estudiantes de las carreras de Pedagogía en Matemática, Castellano e Historia de las zonas norte, centro y sur de Chile. Se utilizó una muestra de 1085 estudiantes universitarios de pedagogía de ambos sexos pertenecientes a universidades del Consejo de Rectores de las Universidades Chilenas. Se empleó un diseño cuantitativo de tipo descriptivo-correlacional. Se aplicaron dos instrumentos: Escala de Actitudes hacia el Endeudamiento y Escala de Discrepancia del yo. Los resultados indican una correlación positiva entre las distintas dimensiones de discrepancia del yo y la actitud hedonista hacia el endeudamiento. En cuanto a la actitud austera hacia el endeudamiento, está correlacionada negativamente con la discrepancia del yo a nivel social. Respecto a diferencias de género, se aprecia que las mujeres son quienes presentan mayor actitud austera. La importancia de indagar este fenómeno remite a la prevención de situaciones de endeudamiento y la promoción de acciones organizadas al adquirir bienes y/o servicios por medio de la consideración de pautas y dinámicas de las sociedades postmodernas, que refuercen, además, el rol modelador de comportamientos financieros de los docentes en sus estudiantes.
\end{abstract}

Palabras clave: educación superior, estudiantes, formación docente, consumo, endeudamiento.

\footnotetext{
${ }_{1}$ Directora del Núcleo Científico Tecnológico en Ciencias Sociales y Humanidades, y Directora del Centro de Excelencia en Psicología Económica y del Consumo (CEPEC), Universidad de La Frontera, Temuco, Chile. Dirección electrónica: marianela.denegri@ufrontera.cl

2 Investigadora del Centro de Excelencia en Psicología Económica y del Consumo (CEPEC), Universidad de La Frontera, Temuco, Chile. Dirección electrónica: c.caro01@ufromail.cl

3 Investigadora del Centro de Excelencia en Psicología Económica y del Consumo (CEPEC), Universidad de La Frontera, Temuco, Chile. Dirección electrónica: m.cerda02@ufromail.cl

4 Investigadora del Centro de Excelencia en Psicología Económica y del Consumo (CEPEC), Universidad de La Frontera, Temuco, Chile. Dirección electrónica: k.eschmann02@ufromail.cl

5 Investigadora del Centro de Excelencia en Psicología Económica y del Consumo (CEPEC), Universidad de La Frontera, Temuco, Chile.Dirección electrónica: n.martinez05@ufromail.cl

6 Investigadora del Centro de Excelencia en Psicología Económica y del Consumo (CEPEC), Universidad de La Frontera, Temuco, Chile. Dirección electrónica: jocelyne.sepulveda@ufrontera.cl
}

Artículo recibido: 5 de junio, 2016

Enviado a corrección: 25 de enero, 2017

Aprobado: 15 de mayo, 2017 


\begin{abstract}
Globalization has affected different social norms and dynamics, emphasizing practices associated with consumption and indebtedness. In this context, Pedagogy undergraduate students are a group of interest due to the fact that they are active citizens and, at the same time, they will be responsible of educating a new generation of citizens. Therefore, the present study aimed to identify the association between demographic, self-discrepancy and psychological variables and the attitude toward indebtedness of undergraduate students of Pedagogy in Mathematics, Spanish and History from the North, Center and South zones of Chile. The sample was composed of 1085 Pedagogy undergraduate students of both genders enrolled in universities belonging to The Chilean Universities Rectors Council. The study used a quantitative design with a descriptive-correlational scope. Participants responded the Scale of Attitudes toward Indebtedness and the Self-Discrepancy Scale. The results showed a positive association between different dimensions of self-discrepancy and hedonist attitude toward indebtedness. Austere attitude toward indebtedness showed a negative association with social self-discrepancy. About gender different, results showed that women reported more austere attitudes. The importance of studying this phenomenon related to the necessity of preventing indebtedness and promoting useful strategies to acquire products and services, considering the regulations and dynamics of postmodern societies.
\end{abstract}

Key words: higher education, students, teacher training, consumption, indebtedness.

\title{
1. Introducción ${ }^{7}$
}

La globalización y los cambios culturales asociados a ella han impactado las dinámicas de relaciones al interior de la sociedad chilena con lo cual se le ha dado un poder inusitado al consumo. Así mismo se le ha otorgado una mayor valoración personal y social ya no solo como un medio de satisfacción de necesidades esenciales o básicas, sino como un referente de definición identitaria tanto a nivel individual como colectivo. De esta forma, el consumo se constituye en el gran articulador de la inclusión social y construcción de identidades personales y sociales (Bauman, 2007; Denegri y Martínez, 2004).

Lo anterior implica que los actos de compra ya no se limitan a la adquisición de bienes por sus características funcionales vinculadas a la satisfacción de necesidades básicas, sino que por el contrario, los objetos de consumo se transforman en portadores de significados sociales, cumplen el rol simbólico de reguladores de estados emocionales, representantes de estatus y moduladores de la identidad, fundamentalmente asociados a la búsqueda de placer y autorrealización (Rodríguez, Otero-López y Rodríguez, 2001).

Como señala el informe de Desarrollo Humano en Chile correspondiente al año 2002, para muchos chilenos el consumo tiene un significado similar al que tenía antes el trabajo, y con ello constituye la cristalización física de la identidad individual, al mismo tiempo que se convierte en un anclaje material del vínculo social (PNUD, 2002). A su vez, se han producido profundos cambios en los valores de la sociedad, la que ha cambiado desde una concepción

\footnotetext{
7 Esta investigación forma parte del Proyecto $N^{\circ}$ 1090179, titulado "Alfabetización Económica y patrones de consumo y endeudamiento en estudiantes de pedagogía: hacia un modelo explicativo financiado por el Fondo Nacional de Ciencia y Tecnología (FONDECYT) de la Comisión Nacional de Ciencia y Tecnología de Chile". Universidad de La Frontera, Temuco, Chile. E-mail: marianela.denegri@ufrontera.cl
} 
de austeridad altamente valorada y vinculada a los valores tradicionales de una sociedad que fue fundamentalmente agraria en sus inicios hacia la búsqueda del hedonismo, la satisfacción inmediata de los deseos y el individualismo propio de los cambios de la modernidad y posmodernidad (Lipovetsky, 2007; Denegri et al, 2014a). Con ello, se ha flexibilizado el concepto y actitud hacia la deuda, esto ha permitido mayor desarrollo económico (mayor consumo), pero al mismo tiempo ha aumentado el riesgo de las operaciones financieras especialmente en las poblaciones más vulnerables como son los sectores de menores ingresos y también los jóvenes (Denegri, Fernández, Iturra, Palavecinos y Ripoll, 1999; McElprang, Haskell y Jenkins, 2005; Norvilitis y Santa María, 2002; Norvilitis, Szablicki y Wilson, 2003; PNUD /Injuv, 2003).

En 2014, la Organización para la Cooperación y el Desarrollo Económicos (OCDE) indica que los hogares chilenos mantenían un nivel de endeudamiento cercano al 57\% del ingreso neto, muy por debajo del promedio exhibido por el resto de los países miembros de la organización, tales como Dinamarca, Holanda o Irlanda, entre otros. No obstante, la capacidad de endeudamiento de las personas depende, entre otras cosas, de su riqueza financiera y real. La riqueza del grueso de los países de la OCDE supera largamente la exhibida por Chile, cuestión que parece explicar, al menos en parte, nuestro indicador de deuda más acotado. Ahora bien, si comparamos a Chile con el resto de los países de la muestra que presentan niveles de endeudamiento similares (Polonia, República Checa y Hungría), nuestro país tiene un nivel de carga financiera menor (Superintendencia de Bancos e Instituciones Financieras Chile, 2015).

Sin embargo, la cuestión del endeudamiento familiar es un tema inquietante en Chile, pues junto con constituir una oportunidad social para muchas familias, esto se ha transformado también en un riesgo, principalmente por la facilidad de los créditos de consumo otorgados por casas comerciales con altas tasas de interés, por medio de lo cual se introduce a la familia en un círculo de deuda del que es difícil salir y que puede aumentar la vulnerabilidad de esta. . Así, en promedio, los hogares destinan un $36 \%$ de sus ingresos mensuales a pagar deudas (Echeverría, 2014). En este mismo orden de cosas, según el informe de Endeudamiento de los clientes bancarios (Sbif, 2015), al segmentar por edad se encuentra que los segmentos de deudores de menor y mayor edad exhiben deudas promedio menores a las de los grupos etarios intermedios. De esta misma forma, la deuda promedio de los hombres es considerablemente mayor a la de las mujeres y la deuda 
promedio de los clientes en regiones es menor a la de los clientes de la Región Metropolitana.

Por otra parte, ya en el año 1972 el Informe "Aprender a Ser. La Educación del futuro" patrocinado por las Naciones Unidas, señalaba que un aspecto clave para el desarrollo de la democracia era lograr que cada ciudadano se convirtiera en

gente consciente del desarrollo, así como un consumidor informado, gracias a un conocimiento real de las leyes, mecanismos y piezas de la vida económica de la nación, de la colectividad local, de la empresa, de los conflictos que en ella tienen lugar, de las fuerzas internas y externas que en él actúan, de los medios que se ofrecen a las diferentes clases económicas para influir sobre el reparto del producto social del trabajo, la productividad, la elección de las inversiones y la planificación [...]. En la escuela, y por todos los medios extraescolares, la educación económica debe convertirse en uno de los elementos esenciales de la conciencia y de la cultura de las masas. (Faure et al., 1972, p. 232)

De esta forma, la capacidad de manejar adecuadamente las finanzas y comprender el funcionamiento de la economía, así como desarrollar actitudes racionales hacia el endeudamiento y hábitos de consumo responsables, constituyen competencias esenciales para todos los miembros de la sociedad, incluyendo a los estudiantes universitarios en general y específicamente a los que participan en los procesos de formación inicial de profesores por el futuro impacto tanto de sus prácticas docentes como por el efecto de modelaje que tiene su comportamiento en los propios educandos (González, Mieres y Denegri, 2014; Walstad y Allgood, 1999).

Según lo planteado, es evidente que las transformaciones socioculturales y económicas han tenido impactos etarios diferenciados, especialmente relevantes en el caso de los jóvenes, dado que por su etapa evolutiva de consolidación de la identidad, el acceso al consumo se transforma en un acceso a símbolos y signos que favorecen la autorrealización y la incorporación a una comunidad (Denegri et al., 2010; PNUD/Injuv, 2003).

Esta etapa se caracteriza por la propensión a la adquisición de estereotipos e ideales conforme a los que marca el mercado, el cual se dirige a los jóvenes como individuos en búsqueda de identidad, de hacer notar sus diferencias y de integrarse socialmente. En este escenario, adquiere relevancia la construcción de la identidad juvenil y especialmente un 
aspecto relevante de ella que se refiere a la consolidación del yo y la percepción de discrepancia entre un yo real e ideal, aspecto constantemente exacerbado por los mensajes que emanan de la sociedad de consumo y especialmente de la publicidad (Bell y Dittmar, 2011; Dakanalis et al., 2015) Estas características son las que facilitan un consumo exacerbado de elementos que modifican la cultura de origen por una más ambiciosa, a la cual se podría llamar como globalizadora (Zamora, 2013).

Ahora bien, interesa profundizar en un segmento específico de jóvenes universitarios conformado por los estudiantes de pedagogía, pues desde la política pública se visualiza la educación como uno de los medios más efectivos para promover la equidad en el acceso a oportunidades de mejor calidad de vida para la población. Lo anterior implica que la atención y tensión se centran hoy en la efectividad de los procesos de formación inicial de profesores, por su impacto probable en el mejoramiento de la calidad de las competencias complejas que debe desarrollar la población escolar para responder a las nuevas necesidades de adaptación.

Al respecto, la importancia de desarrollar investigaciones de modo sistemático en la formación y comprensión económica y financiera de los profesores, cobra fuerza si se consideran a lo menos dos factores. En primer lugar, en estudios tanto nacionales como latinoamericanos se observa que el profesorado presenta un alto índice de desgaste profesional ("Burnout") vinculado tanto a condiciones propias de su quehacer como a factores más generales de la situación vital. A ello se le asocia como factor relevante, la situación económica del profesorado y sus hábitos de consumo, lo que generaría un ciclo de endeudamiento, exceso de trabajo para obtener un salario que permita cubrir necesidades, deterioro de la salud y con ello más endeudamiento. Una investigación realizada a profesores chilenos del año 2009 encontró que se presentaba más desgaste psíquico y culpa entre los docentes (Burnout), cuando había mayor preocupación por el Bajo Salario (Caballero et al., 2009). Se constituyen así los profesores en un grupo altamente vulnerable ante las presiones de la sociedad de consumo (Unesco, 2005). En segundo lugar, dentro del ámbito escolar, quienes de modo natural debieran conducir los procesos de aprendizaje orientados a una adecuada alfabetización económica de los estudiantes son precisamente los profesores (Denegri et al., 2014a).

Si bien, existe evidencia acerca de las actitudes hacia el endeudamiento y los perfiles diferenciales de estudiantes universitarios en torno a ello (Denegri, Cabezas, Del Valle, González y Sepúlveda, 2012), no se ha estudiado la relación entre la discrepancia del yo y 
las actitudes hacia el endeudamiento en la edad juvenil y adulta temprana. Esto, a pesar de que esta es la fase de vida en que las personas se vuelven agentes económicos independientes (Burgoyne, Lea, Webley y Young, 1997). Es por ello que resulta relevante investigar esta variable poco indagada en la sociedad chilena y sudamericana y, en específico, en universitarios, por el aporte teórico y empírico en la comprensión del comportamiento económico.

Según la RAE, el concepto "endeudamiento" alude al conjunto de compromisos de pago contraídos por una persona, empresa o nación (Troncoso y Zúñiga, 2014). Las actitudes hacia el endeudamiento son conceptualizadas como las disposiciones más o menos positivas a considerarle como una vía válida para satisfacer las necesidades y deseos de consumo (Denegri et al, 2012). A su vez, la discrepancia del yo se define como la diferencia resultante entre la identidad real de una persona y su identidad ideal (Dittmar y Bond, 2010; Higgins, 1987). De esta forma, es posible hipotetizar que a mayor discrepancia del yo, en términos de la distancia entre mi yo real y mi yo ideal, podría existir una mayor tendencia hacia el endeudamiento como una forma de adquirir bienes o servicios que permitan disminuir esa brecha.

Según Denegri, García, González y Sepúlveda (2014b), los estudiantes de pedagogía resultan esenciales en la formación de personas y entes económicos, pues su desempeño como educadores comprende contenidos que abarcan educación económica y financiera dispuesta en el currículum de educación secundaria en Chile, por ende, es necesario conocer las actitudes que poseen los estudiantes de pedagogía ante el endeudamiento y cómo estas se vinculan con su identidad personal y social.

De esta forma, la presente investigación se formuló la siguiente pregunta ¿Cuál es la relación que existe entre endeudamiento y discrepancia del yo en estudiantes universitarios del norte, centro y sur de Chile?

A su vez, se plantea como objetivo general establecer la asociación entre variables demográficas, psicológicas, y la actitud hacia el endeudamiento en estudiantes de las carreras de Pedagogía en Matemática, Castellano e Historia de tres zonas del país: norte, centro y sur. Y como objetivos específicos:

1) Determinar el grado de vinculación entre la variable discrepancia del yo y las actitudes hacia el endeudamiento.

2) Identificar la relación entre el género y la actitud hacia el endeudamiento en estudiantes universitarios. 
3) Identificar la relación entre el nivel socio económico y la actitud hacia el endeudamiento en estudiantes universitarios.

4) Identificar la relación entre zonas del país y la actitud hacia el endeudamiento en estudiantes universitarios.

\section{Marco Teórico}

A partir de la década de 1980 en Latinoamérica, y producto del contexto de globalización y de la instalación de un modelo económico neoliberal, se han experimentado cambios a nivel productivo, tecnológico, institucional y social que han propiciado el desarrollo de un contexto sociopolítico y económico cultural extremadamente complejo. Ante dicho contexto, la ciudadanía ha debido aprender a adaptarse a cambios orientados a la privatización de las empresas públicas estatales, disminución del gasto público, privatización de las jubilaciones, apertura del capital internacional y abolición de las barreras aduaneras que proporcionan un mercado de ofertas prácticamente inagotables (Torres, 1998; Gempp et al., 2007). De forma particular, en Chile, ello ha traído consigo cambios no solo en el plano económico, sino también en las pautas culturales y en la dinámica relacional establecida entre los individuos, otorgándole un poder inusitado a prácticas como el consumo, en donde su valoración no solo se refiere a la satisfacción de necesidades básicas, sino también como referente de definición identitaria, representando estatus y valía personal y social (Bauman, 2007), lo cual ha impactado en la construcción de la identidad personal y social de los individuos (Denegri y Martínez, 2004).

Así, el modelo económico neoliberal y su implantación ortodoxa en Latinoamérica, especialmente en Chile, más que una doctrina o una simple colección de medidas económicas, constituyó una verdadera corriente civilizatoria propia del capitalismo avanzado, dominado por el capital financiero y sustentado sobre la revolución tecnológica acaecida en la segunda mitad del siglo XX. Esta corriente civilizatoria tiene como centro de gravitación la cultura del consumo, como definitoria en el perfilamiento de las identidades sociales contemporáneas (Fernández, 2008). Desde esta perspectiva, el consumo y sus rituales asociados se constituye tanto un vehículo de construcción de identidades ideales como una forma de relacionarse con otros (Oyserman y Destin, 2010; Elliott y Nam, 2012). En el caso de los jóvenes, ello ocurre en un contexto evolutivo en el que se consolida la identidad y están madurando tanto las destrezas de autocontrol como las percepciones, actitudes y convicciones, por ende, los jóvenes son más susceptibles al poder del mercado (Denegri, García, González y Orellana, 2014b). 
Una característica propia de este escenario económico y cultural es la emergencia de una cultura de sobreconsumo y por ende de endeudamiento (Chien y DeVaney, 2001), favorecida, por una parte, por una mayor facilidad para el acceso al crédito, y por otra, por la instalación en la población de actitudes de mayor tolerancia y aceptación del endeudamiento, como una forma de acceso hedónico a aquellos bienes y servicios anhelados (Denegri, 2007). De esta forma, el uso del crédito y el endeudamiento se convierten en elementos constitutivos de los procesos de socialización económica familiar y social, presentándose en segmentos de consumidores cada vez más jóvenes (Amar, Abello, Denegri y Llanos, 2007; Denegri, Palavecinos, Gempp y Caprile, 2005; Descouvières, 1998).

Según Luna (1998), entre las variables que explicarían esta situación destacan el apoyo social a la deuda, la comparación social, el tipo de locus de control y las actitudes y estilos de manejo del dinero. Desde la perspectiva de la comparación social, los individuos consumen de acuerdo con sus percepciones de la norma para su grupo de referencia. Si la comparación social es con grupos que están sobre el estatus socioeconómico real, esta puede ser una variable subyacente que lleve a los individuos a gastar, incluso, más allá de sus recursos monetarios para adquirir productos que ellos consideran propios del grupo de su aspiración. En consecuencia, si tienen altos ingresos en relación con su grupo de referencia, tenderán a ahorrar el excedente; por el contrario, si cuentan con unos ingresos relativamente bajos en relación con su grupo de referencia, se mostrarán más dispuestos a contraer deudas (Wärneryd, 1999).

Esto acompañado de la facilidad para optar al crédito y las actitudes sociales de aceptación del endeudamiento han creado un ambiente en el que muchos consumidores creen que es imposible sobrevivir si no se adquieren deudas (Avard, Manton, English y Walker, 2005; Lea, Webley y Levine, 1993).

Particularmente, en nuestro país a partir de las medidas de apertura económica tomadas por el Estado en la década de los años ochenta se ha desatado un desarrollo económico acelerado que ha llevado a que la población experimente nuevas realidades económicas, así como un desarrollo material y socioeconómico. Ello se refleja, por ejemplo, en el informe entregado por la Superintendencia de Bancos e Instituciones Financieras en el año 2014 y 2015, el cual señala que las cifras de endeudamiento en el sistema bancario chileno ascienden a 7,6 y 4,5 millones de pesos para hombres y mujeres respectivamente. Del monto total de deuda, un $74 \%$ corresponde al género masculino, mientras que el $26 \%$ 
corresponde al femenino. Respecto a los deudores, el 62\% corresponde a hombres y el $38 \%$ a mujeres.

Ahora bien, un segmento etario que ha sido particularmente sensible al endeudamiento es el de los jóvenes, ante esta situación, el Instituto Nacional de la Juventud de Chile (Injuv) ha estado desarrollando diversos estudios sobre endeudamiento en población juvenil. El estudio del año 2007 reveló que un 33,18\% de los jóvenes entre 15 y 29 años se encuentran endeudados, de los cuales un $26,72 \%$ se encontraba estudiando ese año. Sin embargo, el estudio no establece el nivel educativo de los jóvenes (Injuv, 2007). En el año 2009, el Injuv encontró que la mitad de la población juvenil chilena (50,6\%) tiene deudas impagas o por pagar, así como el 45\% asegura no tener deudas. En este sentido, la proporción de jóvenes con deudas desciende, significativamente, en el caso de créditos para la educación (16,1\%) y créditos hipotecarios (3,5\%), siendo las mujeres de los niveles socioeconómicos medios (C2 y C3), de sectores urbanos y de nivel técnico quienes presentan un mayor nivel de endeudamiento (Injuv, 2009). Recientemente, según el estudio titulado "Endeudamiento en Jóvenes" realizado por el Instituto (2013), un 37\% de los jóvenes encuestados tiene una deuda impaga o por pagar, siendo las mujeres y los jóvenes de sectores socioeconómicos medios los que tienen mayor nivel de endeudamiento. Estos hallazgos se mantienen similares en el estudio realizado por el mismo Injuv en el año 2014 , donde el $38 \%$ de estos se encuentran endeudados, manteniéndose la tendencia de un mayor endeudamiento de las mujeres respecto a los hombres. De este porcentaje de endeudados, un $43 \%$ corresponde al nivel alto, un $36 \%$ al nivel bajo y un $34 \%$ al nivel medio. El $42 \%$ se concentra en la zona centro, le siguen la zona sur con un $38 \%$ y finalmente la zona norte con un $35 \%$. Se observa también, un mayor porcentaje de endeudamiento en el tramo etario de 25 a 29 años (Injuv, 2014).

Particularmente, en el ámbito de educación superior, en el año 2003 Gebaüer, Schafer y Soto (2003) llevaron a cabo un estudio sobre compra y endeudamiento en jóvenes universitarios, donde se señala que un $25 \%$ de los estudiantes universitarios manifiesta realizar compras impulsivas. A su vez, 3 de cada 10 jóvenes están endeudados, de ahí que un 33\% de los jóvenes entre 15 y 29 años alcance la mayoría, ellos pertenecen a los sectores medios y bajos de la sociedad; Por su parte, las mujeres se encuentran levemente más endeudadas (54\%). La mayoría de los jóvenes endeudados poseen algún instrumento financiero $(72 \%)$, por lo que su obtención pasa a ser determinante al momento de adquirir una deuda. 
En relación a esto, según Denegri, Cabezas, Páez, Sepúlveda y Vargas (2009) los campus de las universidades se han convertido en el lugar perfecto para localizar y ofrecer los más variados instrumentos de crédito (tarjetas bancarias y comerciales, y cuentas corrientes con línea de crédito) mientras se observa una absoluta ausencia de esfuerzos educativos hacia la alfabetización económica y financiera, que sumado a la imposibilidad de una remuneración estable aumenta la posibilidad de caer en mora.

Ante este escenario, resulta interesante analizar los elementos que se encuentran en la base de este fenómeno social. En este sentido, es factible indagar sobre la actitud hacia el endeudamiento como variable medular del proceso. Una actitud es la forma como pensamos, sentimos y actuamos frente a algún aspecto de nuestro entorno (Hawkings, Best y Coney, 1994). Con respecto a la Actitud hacia al Endeudamiento, esta se define como la posición subjetiva que un individuo adopta respecto al uso del crédito para la satisfacción de sus necesidades materiales, es decir, la predisposición a responder ante el endeudamiento de manera característica, ya sea mostrando una aceptación hacia el crédito como un aspecto central de los patrones de consumo, o por el contrario, calificándolo como una conducta económica evitable (Contreras, De la Fuente, Fuentealba, García y Soto, 2006). Desde hace algunos años, las actitudes hacia el endeudamiento han ido cambiando desde el rechazo general al endeudamiento hasta la aceptación del crédito como parte de la sociedad moderna de consumo (Zuckerman, 2000). En este campo, la literatura provee evidencia de que los individuos que tienen actitudes más positivas hacia el crédito tienden a usar con más frecuencia el endeudamiento como una forma de satisfacer sus necesidades de consumo (Chien y DeVaney, 2001; Godwin, 1997; Kim y DeVaney, 2001).

Durkin (2000) sugiere que el desarrollo de estas actitudes está influenciado por las experiencias de consumo, tanto directas como vicarias, y no solo por la experiencia concreta del individuo. Al respecto, Denegri et al. (1999) identifican dos perfiles o estilos actitudinales: el "Austero" y el "Hedonista". El primer estilo se caracteriza por un enfrentamiento cauteloso al endeudamiento y el mantenimiento de una posición más tradicional en el manejo y administración de los recursos financieros. De forma contraria, el segundo estilo corresponde al uso del endeudamiento como recurso habitual para el logro de los bienes y servicios requeridos.

Otra variable importante la constituyen los procesos de construcción de identidad en la juventud, los cuales tienden cada vez más a desligarse de las maneras tradicionales de construir sus referentes (memoria histórica, espacio territorial delimitado, experiencias de 
vida como comunidad, tiempo cronológico), con ello emerge el valor simbólico de los objetos como un nuevo e importante referente de identificación y diferenciación por el significado que los grupos de jóvenes les confieren (Martin-Barbero, 2002; Lipovestky, 2000).

En este proceso de construcción de la identidad juvenil, la consolidación del autoconcepto y especialmente de sus dimensiones reales e ideales se constituye en un elemento gravitante que determinara tanto la disposición como el comportamiento del individuo frente a la toma de decisiones de consumo. Al respecto, la discrepancia del yo se refiere al grado de discordancia de un individuo entre lo que le gustaría ser y lo que es (Dittmar y Bond, 2010; Higgins, 1987). Según Dittmar (2005), el Yo estaría compuesto por siete dimensiones: intelectual, que hace referencia a las capacidades cognitivas como la inteligencia y la memoria; dimensión física, que se asocia a la satisfacción que presentan los individuos con sus cuerpos y cómo se sienten en ellos, esto reflejado en el peso y la capacidad deportiva; dimensión belleza física, en cuanto a sentirse atractivo para sí mismo y los demás en las primeras impresiones visuales; dimensión personal, que hace referencia a si el individuo se siente conforme como persona en todos los ámbitos de su vida; dimensión social, ligada al ajuste que presentan los sujetos en la relación con la familia, los amigos y el entorno en general; dimensión emocional, vinculada a la percepción que tienen los individuos de sus sentimientos y emociones; y por último, la dimensión económica, asociada a la adquisición y al valor que se le otorga al dinero, estatus y prestigio (Luna-Arocas, Puello y Botero, 2004), que en su conjunto se construyen como dimensiones que afectan a una unicidad global expresada en la identidad personal y social, y organizada en el self (Botero, Gutiérrez, Manjarrés y Torres, 1998; Luna-Arocas, Puello y Botero, 2004).

En relación con esto, la teoría de la compensación simbólica del Yo (Wicklund y Gollwitzer, 1982) plantea que las personas usan los recursos materiales para compensar los déficit o discrepancias percibidas en su Yo e identidad. Una de las consecuencias directas se observa en la compra impulsiva, donde las personas pueden ser impulsivas dependiendo del momento y la vinculación con el objeto deseado.

En este marco, y en consideración con lo señalado tempranamente por Katona (1975) cuando analizaba las características de las sociedades de consumo, es casi imposible no contar con una hipoteca, un crédito de consumo o una tarjeta de crédito. La actitud hacia el endeudamiento y su aceptación o rechazo son variables que mediarían la "naturalización" del endeudamiento como un evento cotidiano, que no se cuestiona. Lo anterior podría llevar al 
desarrollo de una cultura de endeudamiento o sobreendeudamiento, lo cual podría implicar peligrosas consecuencias sociales y económicas (Denegri, 2007).

Al respecto, investigaciones previas de este concepto en población universitaria indican correlaciones significativas entre las dimensiones de discrepancia del Yo físico, intelectual y económico, y las actitudes impulsivas y compulsivas hacia la compra; así como en las dimensiones de valores materiales vinculadas al éxito social y felicidad personal. Por ello, es factible inferir que también este constructo podría estar relacionado con las disposiciones actitudinales hacia el endeudamiento, dada su relación con la forma de adquisición de bienes (Denegri, García, González y Sepúlveda, 2014b).

En síntesis, es el proceso globalizador implícito en la modernidad el que ha afectado la construcción de identidades en la medida que se ha acelerado el ritmo de cambio en toda clase de relaciones: "esto hace que sea más difícil para el sujeto comprender lo que está ocurriendo, ver la continuidad entre pasado y presente, y por ende formar una visión unitaria de sí mismo y saber cómo actuar" (Larraín, 2004, p. 62). Ese carácter efímero de los actuales procesos sociales que intervienen en la vida social del hombre moderno Bauman (2006) lo expresa en términos de "vida líquida", como todo aquello que manifiesta lo cambiante del espacio urbano, de las prácticas de consumo, de la cultura, de los gustos, etc.

A decir del autor: "la sociedad moderna líquida, es aquella en que las condiciones de actuación de sus miembros cambian antes de que las formas de actuar se consoliden en unos hábitos y en rutinas determinadas" (Bauman, 2005, p. 9). Se trata, desde esta perspectiva, de una vida dominada por los objetos de consumo y su desechabilidad, por cuanto son mercancías que reportan utilidad efímera e inmediata. La problemática estaría centrada en las consecuencias para la construcción de identidades personales de ese vivir líquido moderno: sin rumbo, sin claridad, veloz en cuanto a cambios, y por supuesto, muy incierto. En este contexto, tal como señala Bauman (2006), la identidad se ha convertido en algo que es auto atribuido, producto de esfuerzos personales y un producto temporal que cambia según los cánones dictados por el mercado. Por lo tanto, el individuo en general y especialmente los jóvenes, estarían en un estado perpetuo de discrepancia del yo, y por lo tanto, en un riesgo permanente de idealizar al endeudamiento como una forma de acceder a aquello que imperiosamente les demanda la sociedad de consumo. 


\section{Metodología}

\subsection{Diseño}

La presente investigación, tras considerar la naturaleza de sus datos, su dimensión temporal y los objetivos, posee un carácter cuantitativo, transversal y descriptivo. Ello implica que se orienta a describir la condición estudiada tal como se manifiesta, para lo cual efectúa una medición en un solo momento y aplica procedimientos estadísticos en el análisis de los datos (Hernández, Fernández y Baptista, 2006; Sousa, Driessnack y Costa, 2007).

\subsection{Participantes}

La población de este estudio comprende a estudiantes universitarios, tanto hombres como mujeres, que se encuentren cursando entre segundo y cuarto año de las Carreras de Pedagogía en Historia, Castellano y Matemáticas, pertenecientes al Consejo de Rectores de las Universidades Chilenas ( $\mathrm{CRUCH}$ ). Se selecciona esta población porque en su ejercicio profesional son los responsables de los contenidos de educación económica y financiera en el Currículum de Educación Secundaria en Chile.

En esta investigación, se utilizó un muestreo probabilístico estratificado garantizando un error tipo I máximo de 0.05 y una potencia de prueba de 0.90 . La muestra quedó constituida por 1085 estudiantes, en donde un $43 \%$ de ellos son hombres y un $57 \%$ son mujeres. La edad promedio fue de 22 años $(D E=2,49)$. Los universitarios pertenecían a las carreras de Pedagogía en Historia 39\%, Castellano 23\% y Matemáticas 38\%. Y tenían residencia en tres zonas de Chile; norte $15 \%$, centro $44 \%$ y sur $41 \%$. Sus estratos socioeconómicos ${ }^{8}$ corresponden a bajo $7 \%$, medio bajo $27 \%$, medio $32 \%$, medio alto $27 \%$, alto $5 \%$ y muy alto correspondiente al $2 \%$.

\subsection{Instrumentos}

\subsubsection{Escala de Discrepancia del yo}

Construida por Dittmar, Beattie y Friese (1996), revisada y adaptada más tarde por Luna-Arocas y Quintanilla (1999), mide el nivel de cercanía de un individuo con sus metas personales. Consta de 7 ítems los que abarcan las dimensiones: "intelectual", "física", "belleza física", "social", "personal", "emocional" y "socioeconómica". Los ítems se encuentran en sentido directo y son de formato tipo Likert con cuatro opciones de respuesta: 1. Como

\footnotetext{
${ }^{8}$ Este aspecto se midió según la Clasificación Socioeconómica (ESOMAR), que cruza las variables nivel educacional y tipo de empleo del jefe/a de hogar (Adimark, 2000).
} 
soy 2. Algo mejor que como soy. 3. Bastante mejor que como soy. 4. Mucho mejor que como soy. Ejemplo de ítem: "A nivel intelectual yo quiero ser algo mejor que como soy (opción 2)". Se entiende que a mayor puntaje existe una mayor discrepancia del Yo. En estudios anteriores este instrumento ha presentado adecuadas propiedades psicométricas, presentando un alpha de Cronbach ( $\alpha$ ) igual a 0,79 (Denegri et al., 2014b).

\subsubsection{Escala de Actitudes hacia el Endeudamiento}

Elaborada por Denegri y cols. (1999), mide la actitud que poseen las personas hacia el endeudamiento. Se pueden distinguir dos dimensiones "Austeridad" (7 ítems) y "Hedonismo" (4 ítems). El primer factor considera actitudes de cautela y reserva, mientras que el segundo evalúa actitudes proclives a contraer deudas sin evaluar las consecuencias. Ambas dimensiones se analizan por separado (Denegri, Cabezas, del Valle Rojas, González, y Sepúlveda, 2012). Los 11 ítems se encuentran en sentido directo y su formato de respuesta es de tipo Likert con 4 opciones que van desde muy de acuerdo hasta muy en desacuerdo. Ejemplo de ítem: "Pedir un préstamo es a veces una muy buena idea". En cuanto a la confiabilidad de este instrumento, estudios realizados por Caamaño, Gallegos, Denegri, Godoy y Cabalín (2012) establecen un alpha de Cronbach total de 0,72, respecto a la sub dimensión Hedonismo $\alpha=0,57$ y a la sub dimensión Austeridad $\alpha=0,89$.

\subsection{Procedimiento}

Los estudiantes fueron invitados individualmente a colaborar en dicha investigación. En primer lugar, se les explicó el objetivo general del estudio y se hizo entrega de un consentimiento informado a través del cual se esclarecen de manera detallada asuntos éticos, tales como la voluntariedad, las consecuencias y beneficios de la participación. Una vez firmado el documento, se procedió a la aplicación de un cuestionario autoadministrado, el que se debía desarrollar de forma individual en aproximadamente 15 minutos. Una vez recogido el material, no hubo entrega alguna de recompensa, no obstante, los participantes tenían la posibilidad de acceder a los resultados de la evaluación si así lo requerían, finalmente se agradeció la colaboración de los estudiantes. 


\subsection{Análisis de datos}

Los datos se analizaron mediante un software estadístico en español, SPSS 16.0. En primera instancia, se evaluó la confiabilidad de cada instrumento, y luego se realizó un análisis factorial exploratorio para la escala de Actitud hacia el Endeudamiento.

Posteriormente, y para dar cuenta de cada objetivo específico se empleó estadística inferencial, se realizaron pruebas que comprenden correlación de variables y diferencia de medias. Se utilizó un nivel de significación de 0,05 para rechazar una hipótesis de nulidad en cada una de ellas.

\section{Resultados}

La presentación de este apartado se organiza de acuerdo con los objetivos específicos propuestos en la presente investigación. Se señalan, en primera instancia, los datos de fiabilidad de las escalas utilizadas, y posteriormente, la descripción de los resultados obtenidos de acuerdo con los análisis estadísticos empleados.

En primera instancia, se evaluó la confiabilidad de cada instrumento. En relación a la Escala Discrepancia el yo, esta obtuvo un alpha de Cronbach total, incluyendo todas sus dimensiones, de 0,78. Por su parte la Escala de Actitud hacia el endeudamiento presentó un alpha total de 0,49 , el cual se consideró insuficiente. Por ello, se decidió realizar un análisis factorial exploratorio, este dio cuenta de tres sub dimensiones que explican el $49 \%$ de la varianza total $\left[\mathrm{KMO}=0,734\right.$; Test de esfericidad de Bartlett: $\left.X^{2}(55)=1612,188, p<0,001\right]($ Ver Tabla 1). 
Tabla 1.

Varianza total explicada

\begin{tabular}{|c|c|c|c|c|c|c|c|c|c|}
\hline \multirow[t]{2}{*}{$\begin{array}{l}\text { Compone } \\
\text { nte }\end{array}$} & \multicolumn{3}{|c|}{ Autovalores iniciales } & \multicolumn{3}{|c|}{$\begin{array}{l}\text { Sumas de las saturaciones } \\
\text { al cuadrado de la extracción }\end{array}$} & \multicolumn{3}{|c|}{$\begin{array}{c}\text { Suma de las saturaciones al } \\
\text { cuadrado de la rotación }\end{array}$} \\
\hline & $\begin{array}{l}\text { To } \\
\text { tal }\end{array}$ & $\begin{array}{c}\% \text { de la } \\
\text { varianz } \\
\text { a }\end{array}$ & $\begin{array}{c}\% \\
\text { acumula } \\
\text { do }\end{array}$ & $\begin{array}{l}\text { Tot } \\
\text { al }\end{array}$ & $\begin{array}{c}\% \text { de la } \\
\text { varianza }\end{array}$ & $\begin{array}{c}\% \\
\text { acumulad } \\
0\end{array}$ & $\begin{array}{l}\text { Tot } \\
\text { al }\end{array}$ & $\begin{array}{c}\% \text { de la } \\
\text { varianza }\end{array}$ & $\begin{array}{c}\% \\
\text { acumulad } \\
0\end{array}$ \\
\hline 1 & $\begin{array}{r}2,4 \\
87\end{array}$ & 22,613 & 22,613 & $\begin{array}{r}2,4 \\
87\end{array}$ & 22,613 & 22,613 & $\begin{array}{r}2,2 \\
29\end{array}$ & 20,261 & 20,261 \\
\hline 2 & $\begin{array}{r}1,8 \\
48\end{array}$ & 16,798 & 39,411 & $\begin{array}{r}1,8 \\
48\end{array}$ & 16,798 & 39,411 & $\begin{array}{r}1,9 \\
23\end{array}$ & 17,484 & 37,745 \\
\hline 3 & $\begin{array}{r}1,1 \\
02\end{array}$ & 10,014 & 49,425 & $\begin{array}{r}1,1 \\
02\end{array}$ & 10,014 & 49,425 & $\begin{array}{r}1,2 \\
85\end{array}$ & 11,680 & 49,425 \\
\hline 4 & $\begin{array}{r}0,8 \\
67\end{array}$ & 7,881 & 57,306 & & & & & & \\
\hline 5 & $\begin{array}{r}0,8 \\
47\end{array}$ & 7,700 & 65,006 & & & & & & \\
\hline 6 & $\begin{array}{r}0,8 \\
14\end{array}$ & 7,400 & 72,406 & & & & & & \\
\hline 7 & $\begin{array}{r}0,7 \\
56\end{array}$ & 6,873 & 79,279 & & & & & & \\
\hline 8 & $\begin{array}{r}0,6 \\
84\end{array}$ & 6,214 & 85,493 & & & & & & \\
\hline 9 & $\begin{array}{r}0,6 \\
43\end{array}$ & 5,844 & 91,337 & & & & & & \\
\hline 10 & $\begin{array}{r}0,5 \\
25\end{array}$ & 4,773 & 96,111 & & & & & & \\
\hline 11 & $\begin{array}{r}0,4 \\
28\end{array}$ & 3,889 & 100,000 & & & & & & \\
\hline
\end{tabular}

Nota. Método de extracción: Análisis de Componentes principales.

Fuente: Elaboración propia basada en varianza explicada de Escala de actitud hacia el endeudamiento. (2015)

Dada la baja carga factorial de algunos ítems se procedió a eliminar tres de ellos (ítems 4, 5 y 10). Por consiguiente, para un análisis más apropiado de los datos, la escala quedó compuesta por 8 ítems en total agrupados en dos dimensiones, cinco ítems referidos al factor austeridad $(\alpha=0,65)$ y otros tres al factor hedonismo $(\alpha=0,63)$, ambos fueron analizados de forma independiente.

Para dar respuesta al primer objetivo específico, se calculó el grado de asociación entre la escala Discrepancia del yo y cada dimensión de la escala Actitud hacia el endeudamiento (hedonismo y austeridad). Se empleó la prueba correlación $r$ de Pearson para dicho análisis, se obtuvo una correlación de carácter positiva para la dimensión de hedonismo $\left(r_{(1085)}=0,091 ; p \leq 0,05\right)$, en cambio, la segunda dimensión, austeridad, no presenta una correlación con la variable Discrepancia del yo $\left(r_{(1085)}=-0,33, p=0,270\right)$. Si bien existe una correlación significativa entre Discrepancia del yo y Actitud hedonista, debido a la muestra de gran tamaño, el efecto del tamaño es mínimo pues el porcentaje de varianza 
compartida no alcanza el 1\% $(0,091)$. Para profundizar en el análisis, se calculó el grado de asociación entre las 7 dimensiones de la escala de Discrepancia del yo y cada dimensión de la escala Actitud hacia el endeudamiento. Los resultados indican que existe asociación significativa positiva entre las dimensiones Discrepancia del yo en belleza física y Actitud Hedonista hacia el endeudamiento $\left(r_{(1083)}=0,069 ; p \leq 0,05\right)$; Discrepancia del yo social y Actitud Hedonista hacia el endeudamiento $\left(r_{(1085)}=0,078 ; p \leq 0,01\right)$; Discrepancia del yo personal y Actitud Hedonista hacia el endeudamiento $\left(r_{(1079)}=0,061 ; p \leq 0,05\right)$; y Discrepancia del yo económico y Actitud Hedonista hacia el endeudamiento $\left(r_{(1084)}=0,147 ; p \leq 0,000\right)$. Además se encontró una relación significativa negativa entre Discrepancia del yo social y Actitud Hedonista hacia el endeudamiento $\left(r_{(1085)}=-0,082 ; p \leq 0,05\right)$.

Para el segundo objetivo específico, se efectuaron dos pruebas $t$ de Student para muestras independientes, la primera de ellas comparó el promedio de hombres y mujeres en relación con su actitud hedonista. Respecto a estos dos grupos, el promedio de los hombres fue de 1,88 $(D E=0,63)$; por su parte, las mujeres presentaron una media de 1,89 $(D E=0,59)$, consecuentemente, no existe una diferencia significativa entre ambos sexos $\left(t{ }_{(1083)}=-0,43\right.$; $p=0,66)$. La segunda de estas pruebas comparó el promedio de hombres y mujeres respecto a la actitud austera. Las mujeres obtuvieron una media de 3,75 $(D E=0,37)$ y los hombres una media de $3,68(D E=0,40)$, con lo cual se evidencia la existencia de una diferencia estadísticamente significativa entre ambas mediciones $\left(t_{(1083)}=-3,04 ; p \leq 0,05\right)$.

En respuesta al tercer y cuarto objetivo específico, se efectuaron pruebas ANOVA de un factor, lo que permitió comparar la actitud hacia el endeudamiento (hedonista y austera) en relación con el nivel socioeconómico (NSE) y posteriormente en relación con las zonas del país. En cuanto a los niveles socioeconómicos, para concretar un análisis más riguroso y homogéneo, se agruparon los niveles socioeconómicos en tres grandes grupos, así se aseguró que los porcentajes analizados fueran similares. Al comparar la actitud hedonista con el NSE, se estableció que no existe una diferencia estadísticamente significativa entre las variables $\left(F_{(1084)}=0,31 ; p=0,73\right)$. Por otro lado, al comparar la actitud austera y el NSE, se comprobó que tampoco existen diferencias estadísticamente significativa entre los NSE y la dimensión austeridad $\left(\mathrm{F}_{(1084)}=1,27 ; \mathrm{p}=0,27\right)($ Ver Tabla 2$)$. 
Tabla 2.

Estadísticos descriptivos nivel socioeconómico dimensión hedonismo y austeridad

\begin{tabular}{lccccc}
\hline Nivel Socioeconómico & \multicolumn{3}{c}{ Hedonismo } & \multicolumn{2}{c}{ Austeridad } \\
\hline & $\mathrm{N}$ & $\mathrm{M}$ & $\mathrm{DE}$ & $\mathrm{M}$ & $\mathrm{DE}$ \\
Bajo y Medio Bajo & 369 & 1,91 & 0,63 & 3,73 & 0,39 \\
Medio & 351 & 1,88 & 0,59 & 3,71 & 0,38 \\
Medio- Alto, Alto y Muy alto & 365 & 1,88 & 0,61 & 3,74 & 0,38
\end{tabular}

Fuente: Elaboración propia basada en estadísticos descriptivos por nivel socioeconómico en dimensiones de la Escala de actitud hacia el endeudamiento. (2015)

Al contrastar las medias de las zonas del país y la actitud hedonista hacia el endeudamiento, se estableció que el promedio de la zona norte fue de 1,79 ( $D E=0,58)$, de la zona centro $1,85(D E=0,61)$ y finalmente, la zona sur con un $1,96(D E=0,61)$, lo cual evidencia diferencias estadísticamente significativas entre las zonas $\left(F_{(1084)}=6,64 ; p \leq 0,05\right)$. Para identificar cuáles son las medias que difieren y controlar de esta forma el error de tipo I, se realizó una prueba post hoc (Bonferroni), cuyos resultados arrojaron que existen diferencias significativas en hedonismo entre la zona norte y sur del país, así como también entre la zona centro y sur del país, pero no existen diferencias significativas entre las zonas norte y centro en hedonismo (Ver Tabla 3).

Tabla 3.

Análisis de varianza post hoc de Bonferroni para el factor Hedonismo

\begin{tabular}{cccccc}
\hline $\begin{array}{c}\text { (I) Zona } \\
\text { geográfica }\end{array}$ & $\begin{array}{c}(\mathrm{J}) \text { Zona } \\
\text { geográfica }\end{array}$ & $\begin{array}{c}\text { Diferencia de medias } \\
(\mathrm{I}-\mathrm{J})\end{array}$ & Sig. & \multicolumn{2}{c}{$\begin{array}{c}\text { Intervalo de confianza al } \\
\text { 95\% }\end{array}$} \\
\cline { 5 - 6 } & & & & Límite inferior & $\begin{array}{c}\text { Límite } \\
\text { superior }\end{array}$ \\
\hline \multirow{2}{*}{ Sur } & Centro & $0,11886^{*}$ & 0,010 & 0,0221 & 0,2156 \\
& Norte & $0,17268^{*}$ & 0,006 & 0,0387 & 0,3067 \\
\hline \multirow{2}{*}{ Centro } & Sur & $-0,11886^{*}$ & 0,010 & $-0,2156$ & $-0,0221$ \\
& Norte & 0,05381 & 0,996 & $-0,0792$ & 0,1868 \\
\hline \multirow{2}{*}{ Norte } & Sur & $-0,17268^{*}$ & 0,006 & $-0,3067$ & $-0,0387$ \\
& Centro & $-0,05381$ & 0,996 & $-0,1868$ & 0,0792 \\
\hline${ }^{*} \mathrm{p}=<0,05$ & & & & &
\end{tabular}

Fuente: Elaboración propia basada en análisis de varianza de prueba post-hoc de ANOVA, para dimensión de Hedonismo. (2015) 
Por último, se realizó el análisis de las zonas con la dimensión austeridad, los resultados revelan que los estudiantes que pertenecen a la zona sur tienen una media de 3,70 $D E=0,38)$; aquellos que viven en la zona centro, una media de 3,73 (DE=0,39) y finalmente, los que pertenecen a la zona norte presentan una media de 3,78 (DE=0,37). Sin embargo, los análisis de la prueba ANOVA revelaron que no existe una diferencia estadísticamente significativa entre las zonas $\left(F_{(1084)}=2,73 ; p=0,065\right)$.

\section{Discusión}

La sociedad de consumo se caracteriza por la producción de bienes de rápida obsolescencia, una inestabilidad de los deseos y la insaciabilidad de las necesidades, con lo cual se aumenta permanentemente la discrepancia entre lo deseado y lo conseguido (Bauman, 2007). En este escenario, la Psicología Económica y los estudios asociados se han convertido en herramientas imprescindibles para comprender el comportamiento del ciudadano consumidor y el papel que juegan las variables psicológicas en él. Dentro de estas variables, las actitudes y, específicamente, las actitudes hacia el endeudamiento y su relación con la discrepancia del yo, constituyeron el foco de esta investigación centrada en los jóvenes universitarios pues se considera que constituyen un segmento de la población que cada vez tiene mayor participación en el escenario social y económico.

Los resultados obtenidos en este estudio dan cuenta de la existencia de una correlación positiva entre la discrepancia del yo y la actitud hedonista hacia el endeudamiento, y pese a que las correlaciones son bajas, su dirección es la teóricamente esperada, lo que podría vincularse con la teoría de la Compensación simbólica del Yo (Wicklund, Robert y Gollwitzer, 1982) que propone que la gente usa los bienes materiales, entre otras estrategias, para compensar las discrepancias percibidas en su Yo e identidad. Esto, además, se ve reforzado si se analizan las dimensiones específicas que se encuentran relacionadas, pues la investigación revela que los jóvenes que puntúan más alto en la dimensión de actitud hedonista hacia el endeudamiento presentarían una mayor discrepancia del yo en las dimensiones física, social, personal y económica.

Estos antecedentes apoyan la idea expuesta sobre la posible compensación simbólica del yo que se provocaría a raíz de la compra y el consumo de productos y servicios que, en este caso, estaría mediada por la actitud a adquirir esos productos y servicios a través del endeudamiento, es decir, la satisfacción inmediata de los deseos tan propia de la modernidad y posmodernidad (Lipovetsky, 2007; Denegri et al, 2014b). De esta forma se va 
articulando una estrecha relación entre el grado de concordancia/discordancia entre lo que nos gustaría ser y lo que realmente somos, y el rol que otorgamos a los objetos de consumo transformándolos en portadores de significados sociales, con un rol simbólico de reguladores de estados emocionales, representantes de estatus y moduladores de la identidad, fundamentalmente asociados a la búsqueda de placer y autorrealización (Dittmar y Bond, 2010; Higgins, 1987; Rodríguez, Otero-López y Rodríguez, 2001).

Esta relación de compensación simbólica sería especialmente fuerte en la etapa juvenil donde una de las principales tareas del desarrollo es la consolidación del proceso de construcción de identidad. En este proceso, el consumo sería el articulador entre la identidad real y la identidad ideal (Zamora, 2013).

Por otra parte, los resultados sobre la relación entre el género y la actitud hacia el endeudamiento revelaron que tanto hombres como mujeres presentan una tendencia similar hacia el endeudamiento hedónico. No obstante, las mujeres presentan una mayor tendencia a presentar actitudes austeras. Si bien, ello contradice el resultado del estudio de Endeudamiento en Jóvenes realizado por el Instituto Nacional de la Juventud (2013) - que revela que las mujeres mantienen una mayor tendencia al endeudamiento en el país - es concordante con los resultados del endeudamiento global de la población, en el cual las mujeres presentan una deuda promedio menor, pero un apalancamiento en la mayoría de los casos superior al de los hombres. Lo anterior se relaciona fundamentalmente con la presencia de brechas de ingreso que desfavorecen a las mujeres.

A pesar de lo mencionado supra, los indicadores de comportamiento de pago y morosidad son mejores en el caso de las mujeres (SBIF, 2014). Debido a esto se hace interesante trabajar a futuro en una investigación que toque más a fondo la diferencia de género en la temática de endeudamiento y discrepancia del yo, principalmente si se toma en cuenta que, según Álvarez y Ruiz-Tagle (2016), generalmente en Chile, las mujeres cuentan con menores niveles de alfabetismo financiero. En cuanto a las diferencias territoriales, los datos de endeudamiento en jóvenes señalan que las regiones del sur de Chile presentan el mayor porcentaje de jóvenes con deuda (50\%) lo que podría relacionarse con una actitud positiva o hedonista hacia el endeudamiento lo que concuerda con los resultados encontrados en esta investigación (Injuv, 2013; 2014) donde las zonas del norte y centro del país tienen una actitud menos hedonista en comparación con la zona sur del país. En cuanto a la actitud austera, todas las zonas del país presentan una tendencia similar. 
En relación con los niveles socioeconómicos y la actitud hacia el endeudamiento, no se encontraron diferencias significativas, por lo tanto todos los niveles socioeconómicos presentarían una actitud similar hacia el endeudamiento. Ello, pese a que las condiciones para acceder a instrumentos financieros son distintas entre un nivel y otro como también lo son sus consecuencias. De forma contraria, un estudio sobre compra y endeudamiento en jóvenes universitarios en Chile (Gebaüer et al., 2003), da a conocer que los jóvenes que se encuentran endeudados son aquellos pertenecientes a los sectores medios y bajos de la sociedad, lo que podría conllevar a establecer que los jóvenes de estos sectores socioeconómicos tienen una actitud más hedonista que austera, en caso de hacer un paralelo de las variables.

De forma similar, el estudio de Endeudamiento y Pobreza en Chile realizado por Echeverría (2014) sobre el endeudamiento familiar arroja que el estrato 1, correspondiente a los deciles de ingreso del 1 al 5, concentra una deuda de 61\%, los deciles de ingreso del 6 al 8 designado como estrato 2 mantiene una deuda $71 \%$, mientras que el estrato 3 , que considera los deciles 9 y 10 evidencia una de deuda de $78 \%$, por lo que existen más estudios que avalan las diferencias entre los estratos económicos que aquellos que reportan similitudes de estos en relación con el endeudamiento.

Se debe tener en cuenta que la población estudiada en esta investigación corresponde mayoritariamente a jóvenes que aún dependen de sus familias, por lo que estas también podrían sopesar en cierta medida aquellos gastos que realicen sus hijos.

Los resultados obtenidos aportan a la reflexión sobre una problemática común a nivel latinoamericano sobre los impactos generales y diferenciados que posee la instalación de un modelo económico y de mercado, el cual orienta a los individuos, y especialmente a aquellos integrantes más jóvenes, a construir sus identidades en torno al consumo (Bauman, 2007). En este escenario, la valoración de la inmediatez en la satisfacción y el abandono al ahorro, por la predominancia del consumo como articulador de la sociedad, naturaliza el endeudamiento como una vía rápida de acceso a los bienes de consumo.

El escenario descrito nos invita a responsabilizarnos por la formación que se está ofreciendo a las generaciones más jóvenes dado que los estudios revelan la falta de educación financiera y económica, que es una variable fundamental para la acción ciudadana responsable (Servicio Nacional del Consumidor, 2015). La formación no solo debería focalizarse en el manejo de contenidos financieros, a la par resulta esencial el desarrollo de destrezas y habilidades financieras, junto con el desarrollo de un pensamiento 
crítico y sistémico, que realmente les posibilite ejercer una ciudadanía plena y consciente de sus derechos y obligaciones como ciudadanos económicos.

Como una limitación propia del carácter de la muestra centrada solo en estudiantes universitarios, es deseable que para investigaciones posteriores se amplíe y diversifique la muestra, de modo que ya no solo se utilice a estudiantes universitarios como sujetos de estudio, sino también a jóvenes que ya se encuentren ejerciendo su profesión, esto, para lograr mayor alcance y observar si existen variaciones significativas.

Cabe destacar que los resultados obtenidos son un aporte dentro de la búsqueda del establecimiento de los orígenes del endeudamiento, lo que deja al descubierto cómo las variables psicológicas son un factor igual de preponderante que aquellos factores de origen social como la vulnerabilidad socioeconómica y el modelo económico que impera en una determinada sociedad, entre otros. A su vez, es información relevante para generar planes de acción y combatir la realidad alarmante en la que se ven envueltos los jóvenes hoy en día. Adicionalmente, resulta de interés, analizar variables como actitudes hacia el dinero y/o el ahorro, percepciones sobre el modelo económico, comprensión de procesos eco nómicos y financieros, entre otras, que permitan avanzar en la comprensión del comportamiento económico.

Se considera imprescindible avanzar en investigaciones orientadas a comprender el funcionamiento de la actual cultura latinoamericana, donde se analice y reflexione en torno a los impactos que ha tenido y continúa ejerciendo el modelo económico instalado en la construcción de los imaginarios sociales (Vázquez, 2005). De igual forma, para futuras investigaciones sería interesante avanzar hacia técnicas de análisis multivariantes, como modelos de ecuaciones estructurales para probar y estimar relaciones causales.

Al respecto, la identidad personal en sociedades de consumo expresaría a la vez elementos de continuidad y de fuertes discontinuidades, donde el yo está sometido a una permanente tensión entre lo real y lo ideal y entre lo posible y lo impuesto por los modelos ideales de un mundo bombardeado por imágenes de cuerpos perfectos, de vidas perfectas (Baudrillard, 2009), de sujetos que satisfacen todos sus deseos a través de mecanismos que constituyen casi talismanes o fetiches como las tarjetas de crédito.

De esta forma, si bien el propio modelo económico neoliberal defiende sustentarse en una concepción del individuo que libre y racionalmente ejerce sus decisiones de consumo, al mismo tiempo, ejerce un sometimiento continuo del individuo al sistema mediante la constante insatisfacción generada por una oferta inagotable de satisfactores de deseos, por 
la exacerbación del hedonismo como medio para lograr acotados momentos de felicidad y por el imperativo de una construcción de identidad continua en un contexto liquido e impredecible (Bauman, 2006; 2007). En definitiva, la objetivación de un sujeto apto para el consumo masivo y auto reproductor del sistema económico y político implícito.

\section{Referencias}

Adimark. (2000). El nivel socioeconómico ESOMAR. Manual de aplicación. Santiago: Adimark GfK.

Álvarez, Roberto y Ruiz-Tagle, Jaime. (2016). Alfabetismo financiero, Endeudamiento y Morosidad de los Hogares en Chile. Serie de documentos de trabajo. Universidad de Chile. Departamento de Economía. Recuperado de http://www.microdatos.cl/Documentos/doctos noticias/626 Docto1.pdf

Amar, José, Abello, Raimundo, Denegri, Marianela y Llanos, Marina. (2007). Pensamiento Económico en jóvenes universitarios. Revista Latinoamericana de Psicología, 39(2), 363-373.

Avard, Stephen, Manton, Edgard, English, Donald y Walker, Janet. (2005). The financial knowledge of college freshmen. College Student Journal, 39(2), 321-339.

Baudrillard, Jean (2009). La sociedad de consumo: sus mitos, sus estructuras. España: Editorial siglo XXI.

Bauman, Zygmunt. (2006). Vida Líquida. España: Editorial Paidós.

Bauman, Zygmunt. (2007). Vida de consumo. Buenos Aires: Paidós.

Bell, Beth y Dittmar, Helga. (2011). Does media type matter? The role of identification in adolescent girls' media consumption and the impact of different thin-ideal media on body image. Sex Roles, 65(7-8), 478-490.

Botero, María Mercedes, Gutiérrez, Marta, Manjarrés, Luz y Torres, Tatiana. (2008). La Relación del SELF con el consumo en hombres y mujeres entre 18 y 24 años, estudiantes de la Universidad del Norte. Psicología desde el Caribe, (21), 1-31.

Burgoyne, Carole, Lea, Stephen, Webley, Paul y Young, Brian. (1997). Introductory notes for the session on Becoming an economic adult. United Kingdom: Department of Psychology. University of Exeter.

Caamaño, Richard, Gallegos, Eduardo, Denegri, Marianela, Godoy, Ma Pía, y Cabalín, Karen. (2012). Perfil de los consumidores del Gran Temuco. Informe Ejecutivo. Centro de Investigación. Recuperado de http://documentslide.com/documents/perfilconsumidores-gran-temuco-informe-ejecutivo-conf-prensa.html 
Caballero Domínguez, Carmen, González Gutiérrez, Orlando, Mercado Anguila, Daniel, Llanos Barrios, Carlos, Bermejo Barrero, Yamid, y Vergel Medrano, Claudia. (2015). Prevalencia del Síndrome de Burnout y su correlación con factores psicosociales en docentes de una institución universitaria privada de la ciudad de Barranquilla. Psicogente, 12(21). Recuperado de http://www.uv.es/unipsico/pdf/CESQT/Externos/2009 Caballero et al.pdf

Chien, Yi-Wen y DeVaney, Sharon. (2001). The effects of credit attitude and socioeconomic factors on credit card and installment debt. The Journal of Consumer Affairs, 35, 179162.

Contreras, Lorena, De la Fuente, Carla, Fuentealba, Jaime, García, Alejandro y Soto, Miriam. (2006). Psicología del Endeudamiento: Una Investigación Teórica. Recuperado de http://docplayer.es/5624400-Psicologia-del-endeudamiento-una-investigacionteorica.html

Dakanalis, Antonios, Carrà, Giuseppe, Calogero, Rachel, Fida, Roberta, Clerici, Massimo, Zanetti, Maria y Riva, Giuseppe. (2015). The developmental effects of media-ideal internalization and self-objectification processes on adolescents' negative bodyfeelings, dietary restraint, and binge eating. European child \& adolescent psychiatry, 24(8), 997-1010.

Denegri, Marianela, Fernández, Francisco, Iturra, Ricardo, Palavecinos, Mireya y Ripoll, Miguel. (1999). Consumir para vivir y no vivir para consumir. Temuco: Ediciones Universidad de la Frontera.

Denegri, Marianela y Martínez, Gustavo. (2004) ¿Ciudadanos o consumidores? Aportes constructivista a la educación para el consumo. PAIDEIA. Revista de Educación, (37), 101-116.

Denegri, Marianela, Palavecinos, Mireya, Gempp, René y Caprile, Cristina. (2005). Socialização econômica em famílias chilenas de classe média: educando cidadãos ou consumidores? Psicología e Sociedade, 17(2), 88 -98.

Denegri, Marianela. (2007). Introducción a la psicología económica. Colombia: PSICOM Editores.

Denegri, Marianela, Cabezas, Daniel, Páez, Alex, Sepúlveda, Jocelyne y Vargas, Mauricio. (2009) Alfabetización económica en estudiantes Universitarios. Calidad en la educación, 30(1), 234-249.

Denegri, Marianela, Cabezas, Daniel, Páez, Alex, Sanhueza, Osvaldo, Vargas, Mauricio, Zapata, Luis y Sepúlveda, Jocelyne. (2010) Actitudes hacia el Endeudamiento en Adolescentes de Educación Municipal y Particular-Subvencionada de la ciudad de Temuco. Educación y Humanidades, 2(1), 47-63.

Denegri, Marianela, Cabezas, Daniel, Del Valle, Carlos, González, Yéssica y Sepúlveda, Jocelyne. (2012). Escala de Actitudes hacia el Endeudamiento: validez factorial y perfiles actitudinales en estudiantes universitarios chilenos. Universitas Psychologica, 11(2), 479-491. 
Denegri, Marianela, Sepúlveda, Jocelyne, González, Tomás, Romero, Gabriela, Ulloa, Jorge y Vásquez, Danilo. (2014a). Actitudes hacia el consumo, compra y materialismo en estudiantes universitarios de pedagogía en Chile. FRONTERAS, 1(2), 45-62.

Denegri, Marianela, García, Constanza, González, Nicolle y Sepúlveda, Jocelyne. (2014b) ¿Educadores o consumidores? Discrepancia del yo, Consumo y valores materiales en estudiantes chilenos de pedagogía. Actualidades Investigativas en Educación, 14(2), 129.

Descouvières, Carlos. (1998). Psicología económica. Santiago, Chile: Editorial Universitaria.

Dittmar, Helga, Beattie, Jane y Friese, Susanne. (1996). Objects, decision considerations and self-image in men's and women's impulse purchases. Acta Psychological, 93, 187-206.

Dittmar, Helga. (2005). La compra compulsiva - ¿una preocupación en crecimiento? Una mirada del género, edad y el apoyo de los valores materialistas. Revista Británica de Psicología, 96(4), 467- 25.

Dittmar, Helga y Bond, Rod. (2010). I want it and I want it now. Using a temporal discounting paradigm to examine predictors of consumer impulsivity. British Journal of Psychology, $101,751-776$.

Durkin, Thomas. (2000). Credit cards: Use and consumer attitudes, 1970-2000. Federal Reserve Bulletin, 623- 634.

Echeverría, Francisca. (2014). Endeudamiento y pobreza en Chile. Recuperado de http://www.ideapais.cl/docs/estudios/16/final 2.0.pdf

Elliott, William y Nam, IISung. (2012). Is Student Debt Jeopardizing the Short-Ternm Financial Health of U.S. Households? Federal Reserve Bank of St. Louis Review, 95(5), 405-424.

Faure, Edgar, Herrera, Felipe, Razzak, Kaddoura, Abdul, Lopes, Henri, Petrovski, Arthur, Rahnema, Majid, y Champion, Frederick. (1973). Aprender a ser. La educación del futuro. Madrid: Alianza Editorial, S. A.

Fernández, Norma. (2008) Introducción. En Instituto de Estudios y Formación de la CTA (Ed.) Refundar el Estado. Posneoliberalismo en América Latina. Emir Sader. Buenos Aires: Instituto de Estudios y Formación de la CTA.

Gebaüer, Alejandra, Schäfer, Lorena y Soto, Eugenia. (2003). Compra impulsiva en estudiantes universitarios con diferente nivel de formación en economía de la Universidad de La Frontera (Tesis para optar al grado de Licenciado en Psicología). Universidad de La Frontera, Temuco.

Gempp, René, Denegri, Marianela, Caripán, Nadia, Catalán, Valentina, Hermosilla, Solange y Caprile, Cristina. (2007). Desarrollo del Test de Alfabetización Económica para Adultos (TAE-A). Revista Interamericana de Psicología, 41(3), 275-284.

Godwin, Deborah. (1997). Dynamics of households' income, debt, and attitudes toward credit, 1983-1989. Journal of Consumer Affairs, 31(2), 303-325. 
González, Yéssica, Mieres, Manuel, Denegri, Marianela y Sepúlveda, Jocelyne. (2014). La enseñanza de nociones básicas de economía en la formación docente. Una propuesta desde la experiencia de dos instituciones de Educación Superior Regional. Profesorado Revista de Currículum y Formación del Profesorado, 3(18), 411-430.

Hawkins, Del, Best, Roger y Coney, Kenneth. (1994). Comportamiento del consumidor. Repercusiones en la estrategia de marketing. Valencia, España: Addison-Wesley Iberoamericana.

Hernández, Roberto, Fernández, Carlos y Baptista, Pilar. (2006). Metodología de la Investigación. México: Mc Graw-Hill.

Higgins, Edward. (1987). Self-discrepancy. A theory relating self and affect. Psychological Review, 94(3), 319-340.

Instituto Nacional de la Juventud (Injuv). (2007). Quinta Encuesta Nacional de Juventud. Recuperado de https://nucleojuventudes.uchile.cl/wp-content/uploads/2016/12/QuintaEncuesta-Nacional-de-JuventudesMIN.pdf

Instituto Nacional de la Juventud (Injuv). (2009). Sexta Encuesta Nacional de Juventud. Recuperado de http://www.injuv.gob.cl/portal/wpcontent/files $\mathrm{mf} /$ sextaencuestanacionaldejuventud.pdf

Instituto Nacional de la Juventud (Injuv). (2013). Endeudamiento en Jóvenes. Santiago, Chile: Injuv.

Instituto Nacional de la Juventud (Injuv). (2014). Sondeo $N^{\circ} 3$ de Educación Financiera. Santiago, Chile: Injuv.

Katona, George. (1975). Psicología de la economía. Buenos Aires: Editorial El Ateneo.

Kim, Haejeong y DeVaney, Sharon. (2001). The determinants of outstanding balances among credit card revolvers. Financial Counseling and Planning, 12(1), 67-78.

Larraín, Jorge. (2004). Identidad y Modernidad en América Latina. México: Editorial Océano.

Lea, Steven, Webley, Paul y Levine, Mark. (1993). The economic psychology of consumer debt. Journal of Economic Psychology, 14, 85-119.

Lipovestky, Gilles. (2000). El imperio de lo efímero (7a. ed.). Barcelona; España: Editorial Anagrama.

Lipovetsky, Gilles. (2007). La Felicidad Paradójica ensayo sobre la sociedad del Hiperconsumo. Barcelona; España: Editorial Anagrama.

Luna, Roberto. (1998). Dinero, trabajo y consumo. Valencia, España: Promolibro.

Luna-Arocas, Roberto y Quintanilla, Ismael. (Setiembre, 1999). Symbolic Consumption and self enhancement. Paper presented at the Ist Conference on Consumption: Consuming markets, consuming meanings. 1-3. Plymouth, UK. 
Luna-Arocas, Roberto, Puello, Shirley y Botero, María. (2004). La compra impulsiva y los valores materiales en los jóvenes (Colombia). Psicología desde el caribe, (14), 1-26.

McElprang, Melissa, Haskell, Janaan y Jenkins, Susan. (2005). Financial attitudes and spending habits of university freshmen. Proceedings of the Academy for Economics and Economic Education, 8(1), 3-6.

Martin-Barbero, Jesús. (2002). Jóvenes: comunicación e identidad. Pensar lberoamérica. Revista de Cultura. Número 0. Recuperado de http://www.oei.es/pensariberoamerica/ric00a03.htm

Norvilitis, Jill y Santa María, Phillip. (2002). Credit card debt on college campuses: causes, consequences, and solutions. College Student Journal, 36, 356-364.

Norvilitis, Jill, Szablicki, Bernard y Wilson, Sandy. (2003). Factors Influencing Levels of Credit-Card Debt in College Students, Journal of Applied Social Psychology, 33(5), 935-947.

Organización de las Naciones Unidas para la Educación, la Ciencia y la Cultura (Unesco). (2005). Condiciones de trabajo y salud docente. Oficina Regional de Educación para América Latina y el Caribe.

Oyserman, Daphna y Destin, Mesmin. (2010). Identity-Based Motivation: Implications for Intervention. The Counseling Psychologist, 38(7), 1001-1043.

Programa de Naciones Unidas para el Desarrollo (PNUD). (2002). Desarrollo Humano en Chile 2002. Nosotros los chilenos: un desafío cultural, Santiago.

Programa de las Naciones Unidas para el Desarrollo (PNUD) / Instituto Nacional de la Juventud (Injuv). (2003). Transformaciones Culturales e Identidad Juvenil en Chile. Santiago de Chile: PNUD.

Rodríguez, Rafael, Otero-López, José, y Rodríguez, Rosa. (2001). Adicción a la compra: análisis, evaluación y tratamiento. Madrid: Pirámide.

Servicio Nacional del Consumidor. (2015).Conocimiento y comportamiento de jóvenes en temas financieros. Informe. Recuperado de http://www.sernac.cl/wpcontent/uploads/2015/08/Educaci\%C3\%B3n-financiera-en-j\%C3\%B3venes -clavepara-un-desarrollo-econ\%C3\%B3mico-saludable-vf.pdf

Sousa, Valmi, Driessnack, Martha y Costa, Isabel. (2007). Revisión de diseños de investigación resaltantes para enfermería parte 1: Diseños de investigación cuantitativa. Revista Latino-am Enfermagem, 15(3), 1-6.

Superintendencia de Bancos e Instituciones Financieras. (2014). Informe de Endeudamiento de los clientes bancarios (Serie Informes). Santiago de Chile. Recuperado de https://www.sbif.cl/sbifweb3/internet/archivos/publicacion 10620.pdf 
Superintendencia de Bancos e Instituciones Financieras. (2015). Informe de Endeudamiento de los clientes bancarios. Serie Informes. Santiago. Recuperado de http://www.sbif.cl/sbifweb3/internet/archivos/publicacion 10992.pdf

Torres, Juan. (1998) El proceso político de las reformas económicas en América Latina. Buenos Aires: Editorial Piados.

Troncoso, Diego y Zúñiga, Gustavo. (2014). Análisis de los patrones de consumo y endeudamiento de las mujeres de la provincia de Ñuble en el marco de las nuevas vinculaciones Rural-Urbana (Memoria para optar al Título de Ingeniero Comercial). Universidad del Bío-Bío, Chillán.

Vázquez, Francisco. (2005). Empresarios de nosotros mismos. Biopolítica, mercado y soberanía en la gubernamentalidad neoliberal. En Javier Ugarte (Comp.), La administración de la vida. Estudios biopolíticos (pp. 73-103). Barcelona: Anthropos.

Walstad, William y Allgood, Sam. (1999). What does college know about economics? American Economic Review, 89(2), 350-354.

Wärneryd, Karl. (1999). The psychology of saving: A study on economic psychology. Cheldenham, England: Rdward Elgar Publishing.

Wicklund, Robert y Gollwitzer, Peter. (1982). Symbolic self-completion. Hillsdale, NJ: Erlbaum.

Zamora, Paola. (2013). La construcción de la identidad en la adolescencia. El reto de apropiarse de un lugar en el mundo. Revista del colegio de ciencias y humanidades para el bachillerato, 6(19), 57-63.

Zuckerman, Gregory. (2000). Borrowing levels reach a record, sparking debate. The Wall Street Journal, 5, 1-18. 